\title{
Fungal Ecology in a Tertiary Neonatal Intensive Care Unit after 16 Years of Routine Fluconazole Prophylaxis: No Emergence of Native Fluconazole-Resistant Strains
}

\author{
Martina Luparia, MD ${ }^{1}$ Francesca Landi, MD ${ }^{2}$ Alessio Mesini, MD ${ }^{2}$ Maria Angela Militello, MD ${ }^{3}$ \\ Paolo Galletto, MD ${ }^{1}$ Daniele Farina, $\mathrm{MD}^{1}$ Elio Castagnola, $\mathrm{MD}^{2}$ Paolo Manzoni, MD ${ }^{1,3}$
}

\footnotetext{
${ }^{1}$ Department of Neonatology and NICU, Sant'Anna Hospital, Torino, Italy

2 Division of Infectious Diseases, IRCCS Istituto Giannina Gaslini,

Genoa, Italy

${ }^{3}$ Division of Pediatrics, Department of Maternal, Neonatal, and Infant

Medicine, Nuovo Ospedale degli Infermi, ASL Biella, Italy
}

Address for correspondence Elio Castagnola, MD, Division of Infectious Diseases, IRCCS Istituto Giannina Gaslini, via Gerolamo Gaslini, 5 16147, Genoa, Italy (e-mail: castagnola.lavoro@gmail.com).

Am J Perinatol 2019;36(suppl S2):S126-S133.

\begin{abstract}
Keywords

- very low birth weight neonate

- fluconazole prophylaxis

- native fluconazole resistance

Objective We analyzed the fungal ecology of a neonatal intensive care unit (NICU) over a period of 20 consecutive years following the introduction of routine fluconazole prophylaxis for all very low birth weight (VLBW; $<1,500 \mathrm{~g}$ at birth) preterm babies. The aim was to detect the possible appearance of any ecological shifts toward the emergence of native fluconazole-resistant (NFR) fungal species.

Study Design This was a retrospective analysis of clinical and microbiological data of VLBW preterm neonates admitted to a large tertiary NICU in Italy from 1997 to 2016 and surviving more than 3 days. Colonization and infection incidence rates, both for fluconazole-sensitive Candida spp and NFR Candida spp, were calculated for each year. We compared the first 4-year period without prophylaxis (1997-2000) with the last 16year period with use of routine fluconazole prophylaxis (2000-2016).

Results Overall, the incidence of fungal colonization significantly decreased after the introduction of prophylaxis (from $43.4 \%$ to $16.5 \%$ ) as well as the systemic fungal infection incidence (from $16 \%$ to $3.7 \%$ ). The proportion of colonization and infection by NFR Candida spp, on the other hand, did not increase, remaining stable throughout the 16 years of exposure to fluconazole. During the prophylaxis period, 42 of 1,172 VLBW neonates were colonized by NFR species (3.6\%), and of them 11 developed a systemic infection $(0.9 \%)$. During the preprophylaxis period, colonization by these particular species affected 11 of 285 VLBW neonates (3.8\%), and a systemic infection involved 4 neonates (1.4\%).

Conclusion Fluconazole prophylaxis is effective in decreasing Candida colonization and systemic infections in preterm neonates in NICU and did not cause emergence or shifts toward NFR Candida spp over a 16-year surveillance period.
\end{abstract}

Invasive fungal disease (IFDs) are a challenging problem in neonatal intensive care units (NICUs), affecting up to $10 \%$ of very low birth weight (VLBW) preterm neonates ${ }^{1}$ and being associated with high morbidity, high mortality, and late neurodevelopmental impairment. ${ }^{2}$ These infections are typi- cally "late-onset" since their acquisition is nosocomial and takes place during the first days after admission in the $\mathrm{NICU}^{3}$ being caused in most cases by the various Candida species. Only a few cases occur through vertical transmission from the mother and can therefore be classified as "early-onset.".
Copyright $\odot 2019$ by Thieme Medical Publishers, Inc., 333 Seventh Avenue, New York, NY 10001, USA. Tel: +1(212) 584-4662. ISSN 0735-1631. 
Diagnosis of IFD is often difficult because of the nonspecificity of the clinical features and the low sensitivity of the available diagnostic techniques (blood culture mainly). ${ }^{4}$ Furthermore, treatment is not always effective in avoiding negative late outcomes since up to $70 \%$ of the treated babies may die or survive with long-term neurodevelopmental impairment. ${ }^{5}$

Due to the aforementioned concerns related to the high burden of morbidity of IFD, strategies of specific prevention with the use of prophylactic fluconazole have been advocated and hence implemented in several NICUs limited to those preterm neonates who are at a higher risk of IFD. Many studies during the past years have shown the effectiveness and the safety of this practice ${ }^{6-8}$; however, so far, the prophylaxis is not yet used in all NICUs in the European Union. ${ }^{9}$

One of the main concerns behind the reluctance of many NICUs to adopt such strategy is the possible development of resistances, namely through the selection of Candida spp that are natively fluconazole-resistant (NFR) or through acquired resistance to fluconazole in those Candida spp that are natively susceptible to fluconazole.

Most of the available data in this area are not conclusive and mainly focused on adult patients. Our group had previously conducted a surveillance study in our NICU, showing no selection of NFR Candida spp after 6 years of routine administration of fluconazole prophylaxis. ${ }^{10}$

This study aims at updating this information by reporting on the fungal ecology and burden of Candida colonization and infection after 10 additional years, hence reporting the ecological outcomes of our NICU over a 16-year continuous period of routine exposure to fluconazole.

\section{Materials and Methods}

The NICU of the Sant'Anna Hospital, Turin, Italy, is a level III unit in the Turin area, with a mean delivery rate of 3,700 live births per year and 500 admissions, of which 80 to 100 are VLBW infants.

Clinical records of VLBW neonates admitted from January 1997 to December 2016 were retrospectively reviewed for the presence of fungal colonization or IFD. Exclusion criteria were survival less than 3 days, incomplete data, and incomplete weekly cultures.

For every eligible neonate, demographic, gestational, and perinatal data; antenatal risk factors; septic episodes; clinical and microbiological culture results; laboratory data; treatments; and outcomes were recorded.

Our main objective was to monitor over the 20-year period the incidence rates of Candida colonization and infection, as well as the proportion of colonization and infections caused by NFR Candida spp.

In doing so, we additionally made a comparison between the preprophylaxis period (1997-2000) and the prophylaxis period (2001-2016).

In our NICU, routine fluconazole prophylaxis for all VLBW neonates was introduced in $2001 .^{8}$

As per routine internal protocols, fluconazole was administered starting from (day of life) DOL 1 as a single dose intravenously or orally, depending on the availability of a venous line and/or on the tolerance of oral feeding. The original regimen was $6 \mathrm{mg} / \mathrm{kg}$ fluconazole every 72 hours in the first week of life and then every 48 hours from the second week until DOL 30 for neonates with birth weight between 1,000 and $1,500 \mathrm{~g}$ and DOL 45 for extremely low birth weight (ELBW; $<1,000 \mathrm{~g}$ at birth) neonates, or until earlier discharge, or until the need for systemic antifungal therapy due to the onset of IFI. This schedule was partially modified during a 15-month period between 2004 and 2005, when approximately onethird of the VLBW neonates received $3 \mathrm{mg} / \mathrm{kg}$ and another onethird did not receive fluconazole, with drug administration beginning on DOL 3: this was because of the involvement of our NICU in a multicenter trial on fluconazole performed with a NICUs network in Italy. ${ }^{8}$ The dosage became then $3 \mathrm{mg}$ for all the infants starting from 2008.

With presumed IFD, fluconazole was suspended, and systemic antifungal therapy with drugs other than fluconazole given empirically until the culture results were available. $^{11}$ Drugs used for treatment were liposomal amphotericin B and micafungin, at the recommended dosages. After the diagnosis of an episode, removal of the central venous catheter was the standard policy for the management of central intravascular lines.

As a standard of care, all VLBW neonates admitted undergo weekly surveillance cultures for Candida colonization. Routine culture surveillance consists of (1) ear canal swab at birth and (2) at least 3 per week of the following: stool or rectal swab, gastric aspirate, nasopharyngeal or endotracheal secretions if intubated, urine samples, and cultures from surgical and mechanical devices when removed (endotracheal tubes, intravascular catheter, drains, and similar devices). Stool, gastric aspirates, surgical, and intravascular devices were collected in sterile containers; respiratory secretions were obtained with an infant mucus sterile extractor kit supplied with two 3.3-mm suction catheters (Vygon, Ecouen, France); skin, ear, and nasopharynx specimens were obtained on swabs (Labobasi SA, Novazzano, Switzerland); and blood drawn for cultures were submitted in dedicated specimens (BacT/Alert PF, bioMerieux Inc., Durham, NC). Urine samples were obtained by sterile urethral catheterization or suprapubic aspiration of the bladder; samples collected from indwelling catheters or from urine bags were not considered for the diagnosis of IFD.

For the identification of fungi, all specimens were inoculated into chromogen culture plates (Albicans ID, Biomerieux Inc.), which permit a rapid Candida. albicans identification through the blue staining of the colonies after 48 hours of incubation at $37^{\circ} \mathrm{C}$. As a policy of our institution, each fungal isolate was speciated. Differently stained colonies were speciated through a miniaturized system of biochemical tests (Vitec Yeast, Biomerieux Inc.). According to relevant literature, $^{12}$ all C. glabrata, C. krusei, and Aspergillus fumigatus isolates were considered as NFR spp. Although the focus of the study was on the speciation and not on the determination of MICs, all C. guiliermondii and C. tropicalis isolates were tested for susceptibility to fluconazole at the hospital's microbiology service at the time of isolation to include or exclude them 
among the NFR isolates. The standardized microbroth dilution assay was used according to the National Committee for Clinical Laboratory Standards recommendations. The interpretative breakpoints of fluconazole resistance were defined as approximately $64 \mathrm{mg} / \mathrm{mL}$, as recommended by the National Committee for Clinical Laboratory Standards. ${ }^{13}$

All fungal isolates from surveillance cultures or significant clinical isolates obtained during the stay in NICU of the enrolled neonates were evaluated. Infants colonized or infected by Candida spp were identified, and among them, those with Candida spp known for their intrinsic, or native, resistance to fluconazole (such as $C$. glabrata, $C$. krusei) were further identified.

Fungal colonization, either acquired or baseline, was defined as at least one positive surveillance culture at any time during the infant's stay in NICU. Baseline fungal colonization was defined as (1) ear canal swab at birth positive for fungi or (2) isolation of fungi from any site during DOL 1 and DOL 2.

A microbiologically documented fungal disease was defined according to the presence of clinical signs or symptoms consistent with infection, together with documentation of a positive culture from either blood, urine (collected by suprapubic sterile puncture or sterile bladder catheterization, or with growth of more than 10,000 fungal organisms $/ \mathrm{mL}$ ), or cerebrospinal fluid. A positive culture from urine collected without sterile procedures (using urine bags or indwelling catheters, or with growth of less than 10,000 fungal organisms $/ \mathrm{mL}$ ) was considered as fungal colonization of urine. These criteria conform to the guidelines of international consensus documents and the recommendations of the Italian Neonatology Society's Fungal Infections Task Force. ${ }^{4}$

\section{Statistical Analysis}

The demographic and clinical data examined are reported as counts (percentages) for categorical variables and as means \pm standard deviation for normally distributed continuous variables. Proportions and continuous variables were compared using Fisher's exact two-tailed test and Student's $t$ test, respectively. Epidemiology was analyzed evaluating the yearly incidence of NFR Candida spp colonization (at least 1 site) and IFI, yearly incidence of fluconazole-sensitive Can- dida spp colonization and IFI, and rate of progression from colonization to infection for NFR Candida spp and for fluconazole-sensitive Candida spp. A comparison of the whole 16year prophylaxis period with the 4-year period before the introduction of prophylaxis was additionally performed. Specifically, the association between IFI/colonization and prophylaxis period (classified as a dichotomous variable: pre-/postfluconazole) was assessed using Fisher's exact test.

Risk ratios and corresponding 95\% confidence intervals were calculated to compare between-group cumulative incidences, and odds ratios were computed. All tests were twotailed, and a $p<0.05$ was assumed to indicate statistical significance. All analyses were run using the SPSS software, version 13.0 (SPSS Inc., Chicago, IL).

\section{Results}

During the study period (42 consecutive months in the preprophylaxis period followed by 192 consecutive months in the fluconazole period), 1,571 infants were admitted to our NICU and survived more than 3 days.

Among them, 104 (28 in the preprophylaxis period and 76 in the fluconazole period) were excluded because of incomplete data or charts or incorrect/ incomplete weekly surveillance cultures. The excluded patients did not differ from the entire cohort in terms of gestational age or birth weight. The final number of enrolled neonates was thus 1,467 (295 in the preprophylaxis group and 1,172 in the prophylaxis group). - Table 1 shows demographic, clinical, and management characteristics of all patients in the two study periods. There were no significant differences between the two periods in terms of the presence of major (including antenatal) risk factors for fungal colonization and systemic infection. The proportion of neonates born through vaginal delivery was lower in the prophylaxis period (26 vs. $39 \%$ ), according to the trend toward admitting more complicated pregnancies and deliveries to our institution.

The overall mortality rate was lower in the prophylaxis period (decreasing from $11.8 \%$ to $5 \% ; p<0.01$ ).

- Table 2 shows the data on the proportions of colonization and IFD episodes, progression rate from colonization to IFD,

Table 1 Summary of demographic and clinical characteristics

\begin{tabular}{|l|l|l|l|}
\hline & Preprophylaxis period, 1997-2000 & Prophylaxis period, 2001-2016 & $p$-Value \\
\hline Sex (male/female) & $149 / 146$ & $597 / 575$ & NS \\
\hline Gestational age (weeks) & $29.8( \pm 3)$ & $29.2( \pm 3)$ & NS \\
\hline Weight (grams) & $1,218( \pm 275)$ & $1,146( \pm 271)$ & NS \\
\hline Vaginal delivery & $39 \%$ & $26 \%$ & 0.04 \\
\hline Mother's preeclampsia & $15 \%$ & $21 \%$ & 0.04 \\
\hline Neutropenia & $10 \%$ & $13 \%$ & NS \\
\hline Intubation (at least one day) & $63 \%$ & $51 \%$ & NS \\
\hline Hyperglycemia & $17 \%$ & $13 \%$ & NS \\
\hline Mortality (nonattributable to fungi) & $11.8 \%$ & $4.5 \%$ & $<0.01$ \\
\hline
\end{tabular}

Abbreviation: NS, nonsignificant. 
Table 2 Proportions of colonization and IFI episodes, progression rate from colonization to IFI, clustered for fluconazole-sensitive and NFR fungal isolates

\begin{tabular}{|c|c|c|c|c|c|}
\hline & Preprophylaxis period & Prophylaxis period & OR & $95 \% \mathrm{Cl}$ & $p$-Value \\
\hline Total colonization & $\begin{array}{l}128 / 295 \\
(43.3 \%)\end{array}$ & $\begin{array}{l}193 / 1,172 \\
(16.4 \%)\end{array}$ & 0.26 & $0.192-0.346$ & $<0.001$ \\
\hline Total IFI & $\begin{array}{l}47 / 295 \\
(15.9 \%)\end{array}$ & $\begin{array}{l}44 / 1,172 \\
(3.7 \%)\end{array}$ & 0.15 & $0.156-0.282$ & $<0.001$ \\
\hline NFR Candida spp colonization & $\begin{array}{l}11 / 295 \\
(3.7 \%)\end{array}$ & $\begin{array}{l}41 / 1,172 \\
(3.4 \%)\end{array}$ & 0.96 & $0.478-1.908$ & 0.858 \\
\hline NFR Candida spp IFI & $\begin{array}{l}4 / 295 \\
(1.3 \%)\end{array}$ & $\begin{array}{l}11 / 1,172 \\
(0.9 \%)\end{array}$ & 0.70 & $0.222-2.198$ & 0.521 \\
\hline Sensitive Candida spp colonization & $\begin{array}{l}117 / 295 \\
(39.7 \%)\end{array}$ & $\begin{array}{l}152 / 1,172 \\
(13 \%)\end{array}$ & 0.22 & $0.166-0.308$ & $<0.001$ \\
\hline Sensitive Candida spp IFI & $\begin{array}{l}43 / 295 \\
(14.6 \%)\end{array}$ & $\begin{array}{l}33 / 1,172 \\
(2.8 \%)\end{array}$ & 0.17 & $0.102-0.288$ & $<0.001$ \\
\hline Col/IFI Candida spp progression rate & $\begin{array}{l}0.36 \\
(47 / 128)\end{array}$ & $\begin{array}{l}0.23 \\
(44 / 193)\end{array}$ & 0.67 & & 0.61 \\
\hline Col/IFI NFR Candida spp progression rate & $\begin{array}{l}0.36 \\
(4 / 11)\end{array}$ & $\begin{array}{l}0.27 \\
(11 / 41)\end{array}$ & 0.59 & & 0.55 \\
\hline
\end{tabular}

Abbreviations: $\mathrm{Cl}$, confidence interval; IFI, invasive fungal infection; NFR, native fluconazole-resistant; OR, odds ratio.

clustered for fluconazole-sensitive and NFR fungal isolates, and comparing the pre- and postprophylaxis periods. - Table 3 shows a year-by-year overview of colonization and infections by Candida spp, whereas - Fig. 1 shows the year-by-year trends of colonization and infection by NFR Candida spp, reported as the number of episodes per year.

Overall, fungal colonization occurred in 321 (23.5\%) of 1,467 VLBW neonates, with incidence rates of $43.4 \%$ (128/ $295)$ and $16.5 \%(193 / 1,172)$ in the pre- and postprophylaxis periods, respectively $(p<0.001)$.

IFD occurred in 91 (6.1\%) VLBW neonates, with a rate of progression from colonization to infection of 0.27 (91/321); 47 episodes occurred in the preprophylaxis period and 44 in the fluconazole years (incidence rates of $16 \%$ and $3.7 \%$, respectively; $p<0.001$ ).

The pathogens responsible for these 44 breakthrough IFD in colonized patients receiving prophylaxis were $C$. albicans (30 cases), C. parapsilosis (8 cases), C. glabrata (6 cases), C, famata (4), C. guilliermondii (4 cases), C. krusei (3 cases) and $C$. tropicalis (1 cases), and Aspergillus fumigatus (1). Noteworthy 13 neonates were infected by two species: 7 by C. albicans and C. parapsilosis, 2 by C. albicans and C. krusei, 1 by $C$. albicans and C. glabrata, 1 by $C$. tropicalis and C. parapsilosis, 1 by C. famata and C. guilliermondii, and 1 by C. famata and C. glabrata.

Candida-attributable mortality was higher in the preprophylaxis period: $5 / 47$ (10.6\%) versus 3/44 (6.4\%). However, there was no significant difference in the survival rates of infants with NFR IFD compared with fluconazole-sensitive IFD. No Candida subspecies were more likely than others to cause invasive disease once the infant was colonized.

In the fluconazole prophylaxis period, colonization by NFR Candida spp remained very infrequent, affecting only $2 \%$ to $6.6 \%$ of all the VLBW infants admitted yearly, with an overall mean incidence of $3.4 \%$ ( 41 neonates out of 1,172 ). Of importance, this incidence did not increase over the 16 years. Also, the incidence of IFD caused by these FR Candida spp did not increase over time, affecting only 11 (0.9\%) VLBW neonates. It is also important to notice how the IFD episodes were never more than two per year.

In comparison with the 4-year period before the introduction of fluconazole prophylaxis, there were no changes in any of the parameters discussed earlier. In fact, colonization by NFR Candida spp in the preprophylaxis period affected 11 (3.7\%) of 295 neonates, and the number of IFD caused by NFR Candida spp was 4 (1.3\%) of 295 . The incidence rates of these two features were similar to those detected during the prophylaxis period (3.4 and $0.9 \%$, respectively).

Microbiological surveillance on colonization and IFD isolates did not show any significant changes in the relative frequencies of the different NFR Candida spp over the years, including C. glabrata. No outbreak of such subspecies occurred in any period. No fluconazole-related adverse effects or reactions were recorded, and none of the patients had to stop the prophylaxis for problems connected to fluconazole exposure.

\section{Discussion}

This study reports the microbiological and ecological outcomes of a large Italian NICU over 16 years of continuous exposure to fluconazole administered as routine prophylaxis to all the VLBW preterm neonates. We wanted to assess whether this practice had any impact on the development of colonization or IFI by NFR Candida spp in our setting.

Our data show that no increase in the absolute number of episodes of colonization or infection caused by those Candida spp that are inherently resistant to fluconazole occurred during the 16-year study period. In turn, relevant benefits were obtained, thanks to this strategy, as shown by the 


\begin{tabular}{|c|c|c|c|c|c|c|c|c|c|c|c|c|c|c|c|c|c|c|c|c|c|}
\hline 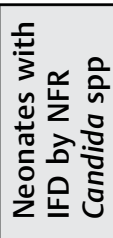 & 0 & 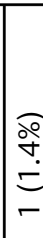 & 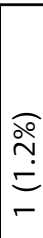 & 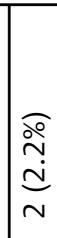 & 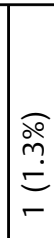 & 总 & 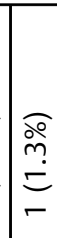 & 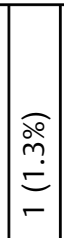 & 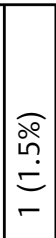 & 0 & 0 & $\begin{array}{l}\stackrel{0}{\circ} \\
= \\
=\end{array}$ & 0 & 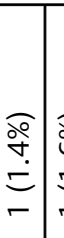 & 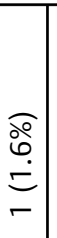 & 0 & 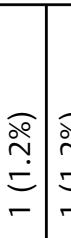 & 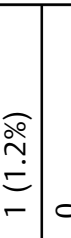 & 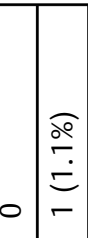 & 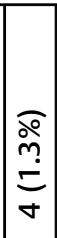 & 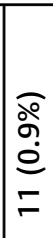 \\
\hline 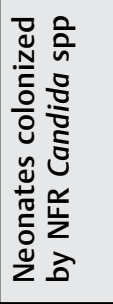 & 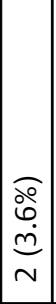 & 兽 & 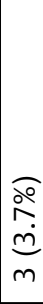 & 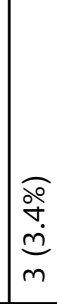 & $\frac{\widehat{\circ}}{\stackrel{\text { dे }}{m}}$ & 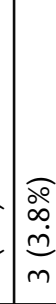 & 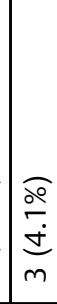 & 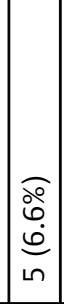 & \begin{tabular}{|l}
$\stackrel{-}{\circ}$ \\
$\stackrel{0}{n}$ \\
\\
$\sim$
\end{tabular} & \begin{tabular}{|l}
$\stackrel{0}{\circ}$ \\
$\infty$ \\
$\grave{d}$ \\
$\sim$ \\
$\sim$
\end{tabular} & 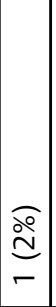 & 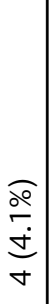 & 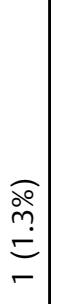 & \begin{tabular}{c|c}
$\stackrel{\circ}{\circ}$ \\
$\stackrel{+}{+}$ \\
$\dot{ \pm}$ \\
$m$
\end{tabular} & $\begin{array}{l}\widehat{\circ} \\
\stackrel{\mathrm{D}}{ \pm} \\
\mathrm{m}\end{array}$ & 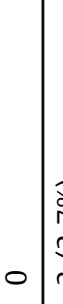 & 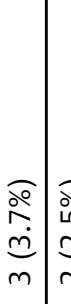 & 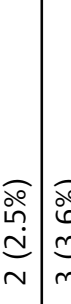 & 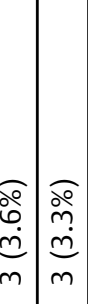 & 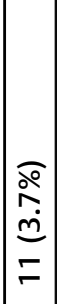 & 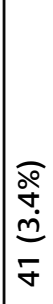 \\
\hline 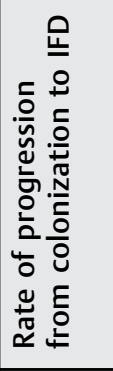 & m. & 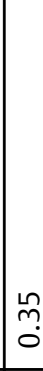 & $\begin{array}{c}\hat{n} \\
0 \\
o\end{array}$ & テ. & $\begin{array}{l}0 \\
0 \\
0\end{array}$ & $\frac{1}{0}$ & 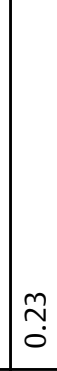 & 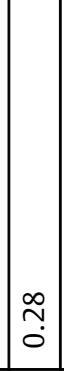 & $\stackrel{2}{0}$ & 0 & \begin{tabular}{|l|}
$\infty$ \\
0 \\
0 \\
\end{tabular} & $\stackrel{\circ}{\check{0}}$ & 0 & 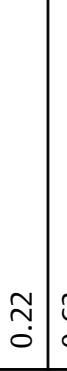 & $\begin{array}{l}m \\
\ddot{0} \\
\dot{0}\end{array}$ & -2 & 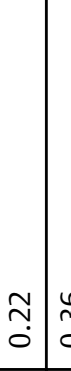 & \begin{tabular}{l|l}
0 & $\vdots$ \\
$m$ & 2 \\
0 & 0 \\
\end{tabular} & \begin{tabular}{c|c}
$\stackrel{n}{N}$ & $\stackrel{n}{0}$ \\
0 & 0 \\
\end{tabular} & \begin{tabular}{|l|}
$\stackrel{n}{n}$ \\
$\tilde{o}$ \\
0
\end{tabular} & $\stackrel{\stackrel{n}{n}}{\stackrel{0}{0}}$ \\
\hline 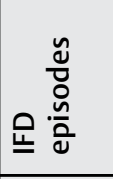 & 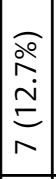 & 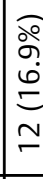 & 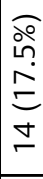 & 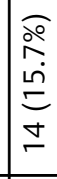 & 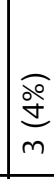 & 总 & 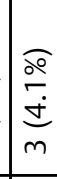 & 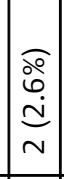 & 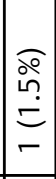 & 0 & 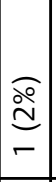 & 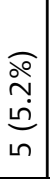 & 0 & 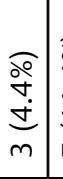 & 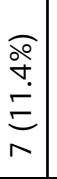 & 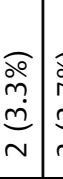 & 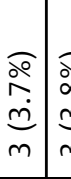 & 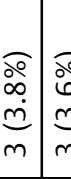 & 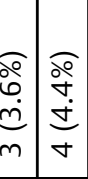 & 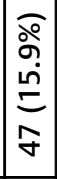 & 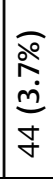 \\
\hline 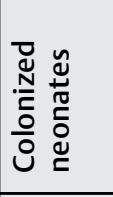 & 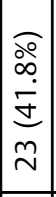 & 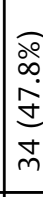 & 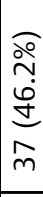 & 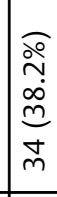 & 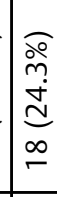 & - & $\begin{array}{l}\widehat{\circ} \\
\infty \\
\frac{\infty}{c}\end{array}$ & 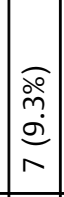 & 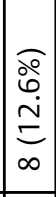 & $\begin{array}{l}\widehat{o} \\
\stackrel{\circ}{+} \\
\infty \\
\infty \\
\sigma \\
\end{array}$ & 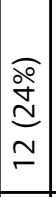 & 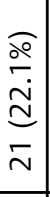 & 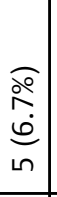 & 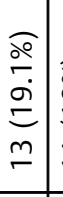 & $\begin{array}{l}\stackrel{\circ}{\circ} \\
\infty \\
= \\
=\end{array}$ & 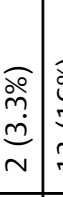 & 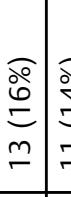 & 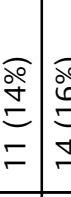 & 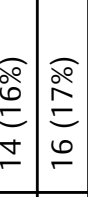 & 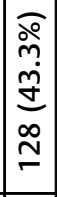 & 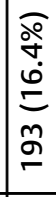 \\
\hline 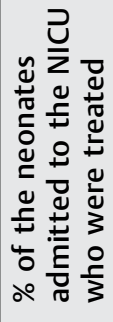 & 0 & 0 & 0 & 0 & $\stackrel{\infty}{\sim}$ & $m$ & $\stackrel{\circ}{m}$ & $\tilde{m}$ & $\stackrel{\varrho}{\sim}$ & $m$ & 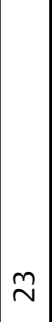 & $\stackrel{\bullet}{\sim}$ & $\stackrel{\sim}{m}$ & $\stackrel{n}{m}$ & $\stackrel{m}{m}$ & $\tilde{m} \mid:$ & $\stackrel{m}{m}$ & \begin{tabular}{l|l}
$\stackrel{m}{m}$ & $\stackrel{n}{m}$
\end{tabular} & $\stackrel{m}{m}) \stackrel{+}{m}$ & 0 & $\bar{m}$ \\
\hline 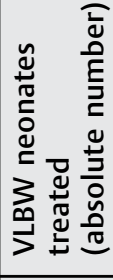 & 织 & $\pi$ & $\infty$ & த & $\stackrel{N}{ }$ & r & $N$ & 迎 & $\ddot{0}$ & $\pi$ & in & 吕 & $\stackrel{\nabla}{\star}$ & $\infty$ & 6 & 周 & \begin{tabular}{l|c}
$\infty$ \\
$\infty$
\end{tabular} & $\begin{array}{c}\wedge \\
\wedge\end{array}$ & $\begin{array}{c}\infty \\
\infty\end{array}$ & 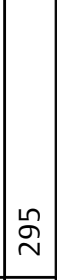 & $\underset{\Sigma}{=}$ \\
\hline 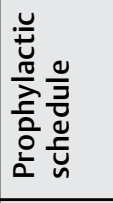 & \begin{tabular}{|l|}
0 \\
$\vdots$ \\
2 \\
$z$
\end{tabular} & $\begin{array}{l}0 \\
\vdots \\
z \\
\end{array}$ & $\begin{array}{l}0 \\
\vdots \\
2\end{array}$ & $\begin{array}{l}0 \\
\text { Lे } \\
2\end{array}$ & Ð & $\stackrel{\bigcup}{己}$ & $\stackrel{\circlearrowright}{己}$ & $\stackrel{U}{\vec{Z}}$ & $\underset{\sqcup}{己}$ & 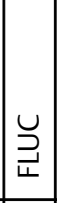 & $\begin{array}{l}\cup \\
\\
\end{array}$ & $\stackrel{\cup}{\supset}$ & $\begin{array}{l}\cup \\
⿱ \\
\\
\end{array}$ & \begin{tabular}{|l}
$\circlearrowright$ \\
\\
\end{tabular} & $\stackrel{\bigcup}{\supset}$ & $\stackrel{U}{\vec{U}}$ & $\stackrel{U}{\ni}$ & $\stackrel{U}{ٍ}$ & 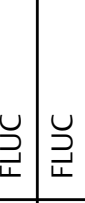 & \begin{tabular}{|l|}
0 \\
$\tilde{0}$ \\
$z$ \\
\end{tabular} & $\stackrel{\bigcup}{\beth}$ \\
\hline ্ָ & I & S. & ন & ¿্ঠे & 오 & ঠे & ڤ̊̀े & 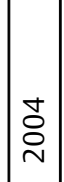 & 总 & 离 & 令 & $\stackrel{\infty}{0}$ & $\begin{array}{l}\stackrel{8}{0} \\
\stackrel{D}{N}\end{array}$ & 号 & $\overline{\bar{N}}$ & \begin{tabular}{l|l}
$\tilde{n}$ & $\vdots$ \\
$\grave{N}$ & $\vdots$
\end{tabular} & \begin{tabular}{c|c}
$m$ \\
$\stackrel{n}{v}$ & $\vdots$ \\
$c$
\end{tabular} & 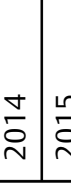 & \begin{tabular}{l|l}
$\mathbf{n}$ & 0 \\
$\grave{n}$ & $\stackrel{0}{N}$ \\
\end{tabular} & 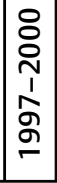 & 定 \\
\hline
\end{tabular}




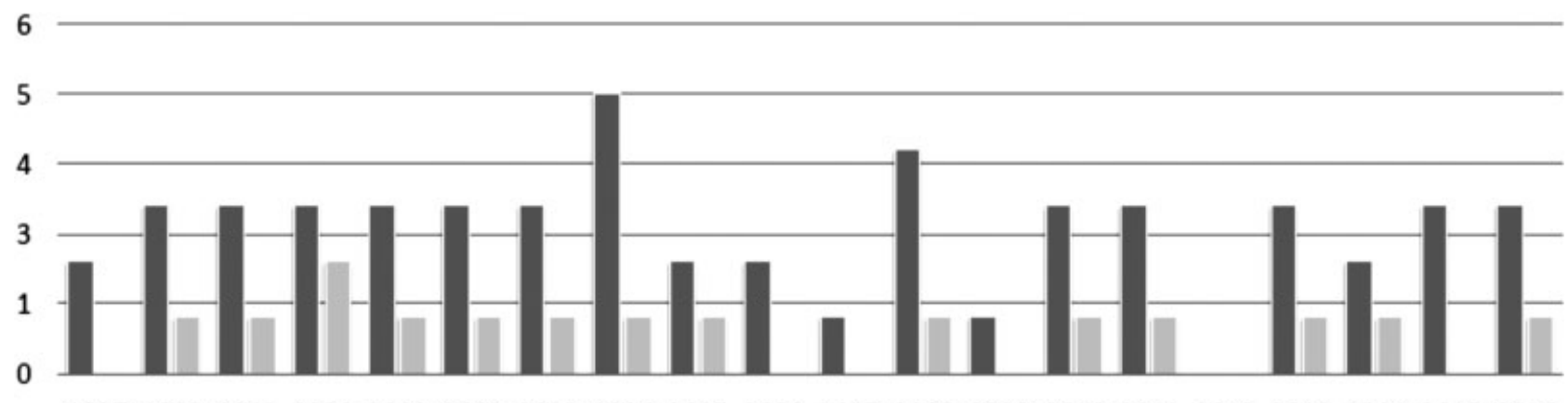

19971998199920002001200220032004200520062007200820092010201120122013201420152016

- NFR Colonizations

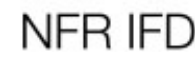

Fig. 1 Year-by-year trend of colonization and infection by NFR Candida spp: number of episodes per year. IFD, invasive fungal disease; NFR, native fluconazole-resistant.

continuous and significant decrease in colonization and infections caused by the Candida spp that are inherently sensitive to this azole.

In other terms, our strategy produced clinically measurable benefits and impacted on the overall fungal ecology of the NICU by only reducing the incidence rates of isolation of Candida spp that are sensitive to fluconazole since these relevant and beneficial changes have not been accompanied by a surge in the morbidity related to the fungal organisms that cannot be tackled by the azole that we used since they are natively resistant to that.

To our knowledge, this is the longest ever ecological and clinical report from a NICU where routine fluconazole prophylaxis is administered.

In general, our data support the view that colonization and infection from NFR species remains a limited and sporadic event when prophylactic fluconazole is used in a NICU. These findings are even more remarkable since our report spans over a very long (16-year) period, during which the total number of colonized or infected neonates by these fungal species remained very low and substantially unchanged.

Our data are consistent with, and add to, what we already observed in our previous 6-year-long study. ${ }^{6}$

Few other studies analyzed this area, with quite similar results, although based on far shorter periods.

Healy et al compared 4 years of fluconazole prophylaxis administered routinely to all the ELBW $(<1,000 \mathrm{~g}$ at birth) neonates in Texas, with the two previous years without prophylaxis, finding no shifts toward resistant Candida spp. ${ }^{14}$ Kaufman et al furthermore demonstrated a slight increase of the minimal inhibitory concentration (MIC) in Candida strains colonizing neonates admitted in a randomized clinical trial of fluconazole prophylaxis. ${ }^{15} \mathrm{~A}$ slightly different experience had been described by Sarvikivi et al in their NICU in Finland: in a 12-year period, they did not detect any increase of $C$. glabrata or $C$. krusei (while using the fluconazole prophylaxis); however, they observed the appearance of resistance in some strains of $C$. parapsilosis, that were originally sensitive to fluconazole. It is important to notice that this event happened when they extended the prophylaxis to all the neonates admitted to the NICU instead of reserving it to high-risk subgroups such as VLBW or ELBW neonates. ${ }^{16}$

Taken together, all the aforementioned data shed a clearer light on the issues and potential concerns related to fungal ecology in a neonatal setting under routine fluconazole exposure. The overall reassuring message becomes even more meaningful when we make a comparison with similar other studies conducted on adults or in nonneonatal settings, which, in turn, demonstrate unwanted changes in resistance patterns. Marr et al showed an association between longterm prophylaxis and emergence of resistant strains in adult patients who underwent bone marrow transplantation. ${ }^{17}$ The same findings were confirmed by Safran and Dawson, who reported a shift toward fluconazole-resistant species in a setting of critical surgical adult patients exposed to routine fluconazole prophylaxis, ${ }^{18}$ and by Husain et al, who described adult patients undergoing liver transplantation. ${ }^{19}$

Some possible explanations can be hypothesized for the differences in the fungal ecological findings in our setting compared with the aforementioned settings of adult patients.

The main difference is that we administered low fluconazole dosages to only selected subgroups of infants for generally shorter periods of time. In fact, each prophylaxized patient received fluconazole doses ranging from 8 to 12 only, according to the twice-weekly schedule and each patient was exposed to a cumulative dose ranging from 24 to $36 \mathrm{mg} / \mathrm{kg}$ only. Finally, the proportion of treated versus admitted patients was always below the $30 \%$ since our NICU admits some 500 patients per year, of which only 100 are, on average, eligible for prophylaxis being VLBW infants.

In contrast, schemes of prophylactic fluconazole administration in adult settings generally include daily doses, with higher cumulative doses, and are delivered to the majority of the patients concomitantly admitted in the unit. The peculiarity of the fluconazole schemes used in the NICU might thus contribute to the phenomenon that we recorded, that is, the absence of detrimental fungal ecological shifts, or, at the 
very least, the fact that possible ecological shifts might need longer periods to occur.

It can be meaningful to remind that in neonatal settings, fluconazole resistances have been described when this practice was expanded to all the patients concomitantly admitted in the NICU, contrary to what happens usually. ${ }^{16}$ The cumulative dose of exposure to fluconazole of the unit, in addition to that of the single individual, might also be of importance in determining the emergence of an ecological shift since recirculation within the unit of such species is likely to occur less frequently when a majority of the patients are not exposed to fluconazole.

This area has been explored by several reports in adult settings ${ }^{20,21}$ and might be a key point in understanding our findings.

Strengths of our study are its length, as it spans over 16 years, and the very high number of neonates included. Based on animal data, it has been suggested that the critical duration of fluconazole exposure needed to detect an ecological shift is 4 years. $^{22}$ Our period of observation is far longer. We therefore speculate that 16 years should be a sufficient time frame to allow the occurrence and detection of possible ecological shifts between the Candida spp subpopulations, also considering the very high number of patients exposed during this period to this prophylaxis.

Nonetheless, we cannot exclude that longer periods of exposure are necessary to promote ecological shifts. Indeed, nobody knows how much time is needed from fluconazole to cause (if ever) a change in fungal ecology in a NICU, especially in a setting like ours, in which the cumulative dose of fluconazole used remained very low compared with that in adult settings.

We acknowledge that our study has limitations. These are retrospective data, coming from a single center, which has specific characteristics that could be different from other centers and that could play a role in the way fungal ecology modifies over time. Moreover, even though the number of enrolled neonates is high, the study does not have enough power to analyze in details what happens in specific subgroups of neonates, for example, in ELBW neonates alone or in neonates with specific additional risk factors, or also what happens in the population of nontreated babies in the NICU. It could be interesting to study in depth the association between NFR Candida infections and the individual characteristics of the single newborn. Finally, we focused on the fungal ecology of different strains of Candida spp in our setting, assessing the relative balance between NFR and notNFR strains, but we did not analyze the MICs of the single Candida spp isolated during the years. Even though very reassuring data have been already published in this area, 23 this additional information will be obviously critical to fully rule out any risk of emergence of resistances to fluconazole in NICU settings.

In conclusion, we think that these data can be helpful in addressing many existing doubts concerning the adoption of prophylaxis in a NICU. Overall, they support the view that there is no risk for emergence of natively resistant strains of Candida spp in NICUs that adopt routine fluconazole pro- phylaxis to selected high-risk groups of neonates during long periods of time.

Continued surveillance and monitoring of the fungal ecology in NICUs where fluconazole prophylaxis is adopted is warranted to exclude that unwanted shifts may occur in longer periods than that reported in this study.

\section{Conflict of Interest}

P. M. received speaker fee from Astellas and E. C. received speaker fees from Astellas and Pfizer. M.L., F.L., A.M., M.M.I., and P.G. have nothing to disclose related to this article.

\section{References}

1 Fridkin SK, Kaufman D, Edwards JR, Shetty S, Horan T. Changing incidence of Candida bloodstream infections among NICU patients in the United States: 1995-2004. Pediatrics 2006;117(05):1680-1687

2 Benjamin DK Jr, Stoll BJ, Gantz MG, et al; Eunice Kennedy Shriver National Institute of Child Health and Human Development Neonatal Research Network. Neonatal candidiasis: epidemiology, risk factors, and clinical judgment. Pediatrics 2010;126(04):e865-e873

3 Stoll BJ, Hansen N, Fanaroff AA, et al. Late-onset sepsis in very low birth weight neonates: the experience of the NICHD Neonatal Research Network. Pediatrics 2002;110(2 Pt 1):285-291

4 Manzoni P, Pedicino R, Stolfi I, et al; Task Force per le infezioni fungine neonatali del GSIN; Socieà Italiana di Neonatologia. Criteria for the diagnosis of systemic fungal infections in newborns: a report from the Task Force on neonatal fungal infections of the GSIN [in Italian]. Pediatr Med Chir 2004;26(02):89-95

5 Benjamin DK Jr, Stoll BJ, Fanaroff AA, et al; National Institute of Child Health and Human Development Neonatal Research Network. Neonatal candidiasis among extremely low birth weight infants: risk factors, mortality rates, and neurodevelopmental outcomes at 18 to 22 months. Pediatrics 2006;117(01):84-92

6 Kicklighter SD, Springer SC, Cox T, Hulsey TC, Turner RB. Fluconazole for prophylaxis against candidal rectal colonization in the very low birth weight infant. Pediatrics 2001;107(02):293-298

7 Kaufman D, Boyle R, Hazen KC, Patrie JT, Robinson M, Donowitz LG. Fluconazole prophylaxis against fungal colonization and infection in preterm infants. N Engl J Med 2001;345(23):1660-1666

8 Manzoni P, Stolfi I, Pugni L, et al; Italian Task Force for the Study and Prevention of Neonatal Fungal Infections; Italian Society of Neonatology. A multicenter, randomized trial of prophylactic fluconazole in preterm neonates. N Engl J Med 2007;356(24):2483-2495

9 Kaguelidou F, Pandolfini C, Manzoni P, Choonara I, Bonati M, Jacqz-Aigrain E. European survey on the use of prophylactic fluconazole in neonatal intensive care units. Eur J Pediatr 2012; 171(03):439-445

10 Manzoni P, Leonessa M, Galletto P, et al. Routine use of fluconazole prophylaxis in a neonatal intensive care unit does not select natively fluconazole-resistant Candida subspecies. Pediatr Infect Dis J 2008;27(08):731-737

11 Benjamin DK Jr, Ross K, McKinney RE Jr, Benjamin DK, Auten R, Fisher RG. When to suspect fungal infection in neonates: a clinical comparison of Candida albicans and Candida parapsilosis fungemia with coagulase-negative staphylococcal bacteremia. Pediatrics 2000;106(04):712-718

12 Kaufman D, Fairchild KD. Clinical microbiology of bacterial and fungal sepsis in very-low-birth-weight infants. Clin Microbiol Rev 2004;17(03):638-680

13 National Committee for Clinical Laboratory Standards. Approved Standard M27-A: Reference Method for Broth Dilution Antifungal Susceptibility Testing of Yeasts. Wayne, PA: National Committee for Clinical Laboratory Standards; 1997 
14 Healy CM, Campbell JR, Zaccaria E, Baker CJ. Fluconazole prophylaxis in extremely low birth weight neonates reduces invasive candidiasis mortality rates without emergence of fluconazoleresistant Candida species. Pediatrics 2008;121(04):703-710

15 Autmizguine J, Smith PB, Prather et al. Effect of fluconazole prophylaxis on candida fluconazole susceptibility in premature infants. J Antimicrob Chemother 2018;73:3482-3487

16 Sarvikivi E, Lyytikäinen O, Soll DR, et al. Emergence of fluconazole resistance in a Candida parapsilosis strain that caused infections in a neonatal intensive care unit. J Clin Microbiol 2005;43(06): 2729-2735

17 Marr KA, Seidel K, White TC, Bowden RA. Candidemia in allogeneic blood and marrow transplant recipients: evolution of risk factors after the adoption of prophylactic fluconazole. J Infect Dis 2000;181(01):309-316

18 Safran DB, Dawson E. The effect of empiric and prophylactic treatment with fluconazole on yeast isolates in a surgical trauma intensive care unit. Arch Surg 1997;132(11):1184-1188
19 Husain S, Tollemar J, Dominguez EA, et al. Changes in the spectrum and risk factors for invasive candidiasis in liver transplant recipients: prospective, multicenter, case-controlled study. Transplantation 2003;75(12):2023-2029

20 Fan X, Xiao M, Liao K, et al. Notable increasing trend in azole nonsusceptible Candida tropicalis causing invasive candidiasis in China (August 2009 to July 2014): molecular epidemiology and clinical azole consumption. Front Microbiol 2017;8:464

21 Marins TA, Marra AR, Edmond MB, et al. Evaluation of Candida bloodstream infection and antifungal utilization in a tertiary care hospital. BMC Infect Dis 2018;18(01):187

22 Yoder BA, Sutton DA, Winter V, Coalson JJ. Resistant Candida parapsilosis associated with long term fluconazole prophylaxis in an animal model. Pediatr Infect Dis J 2004;23(07):687-688

23 Autmizguine J, Smith PB, Prather K, et al; Fluconazole Prophylaxis Study Team. Effect of fluconazole prophylaxis on Candida fluconazole susceptibility in premature infants. J Antimicrob Chemother 2018;73(12):3482-3487 